\title{
Nuclear Suppressors of the Mitochondrial Mutation oxi1-V25 in Saccharomyces cerevisiae. Genetic Analysis of the Suppressors: Absence of Complementation between Non-allelic Mutants
}

\author{
By M. BOGUTA, T. ŻOŁĄDEK AND A. PUTRAMENT* \\ Institute of Biochemistry and Biophysics, Polish Academy of Sciences, Rakowiecka 36, 02-532, \\ Warsaw, Poland
}

(Received 2 December 1985; revised 18 March 1986)

\begin{abstract}
Ten informational nuclear suppressors of the oxil- mitochondrial mutation of Saccharomyces cerevisiae are recessive. They are linked to each other, but their allelism is uncertain. Some of them unfavourably affect functions of standard $\left(\mathrm{mit}^{+}\right)$mitochondrial genomes. One suppressor severely impairs or entirely prevents mitochondrial functions of the spore clones carrying it. The spectrum of mit $^{-}$mutations on which these suppressors act is similar to that exhibited by nam3-1. In double heterozygotes am $_{\mathrm{x}} / \mathrm{NAM}^{+}, \mathrm{NAM}_{\mathrm{x}}^{+} /$nam3-1 $^{-1}$ the oxi1- (and box3-) mutation is suppressed, yet one of our suppressors (R705) and nam3-1 show independent segregation in tetrads. This indicates that there may be absence of complementation between non-allelic suppressors.
\end{abstract}

\section{INTRODUCTION}

Our earlier paper (Żołądek et al., 1985) described the phenotypes of 10 nuclear suppressors of the mitochondrial mutation oxil ${ }^{-}$(UAA) of Saccharomyces cerevisiae (see Methods for details of the nomenclature of mutant alleles). It was suggested that suppression resulted from an alteration in a mitoribosomal protein of the small subunit. Our suppressors proved to be very similar to the nuclear nam 3 and mitochondrial mim 3 suppressors studied by Kruszewska \& Słonimski $(1984 a, b)$ with respect to the spectrum of suppressed mit ${ }^{-}$mutations. For protein synthesis in the yeast cytosol and in Escherichia coli informational suppression resulting from alterations in ribosomal proteins can be due to mutations in any one of two or three genes (Hawthorne \& Leupold, 1974; Ono et al., 1981; recent review: Surguchov et al., 1984). Thus, it was of interest to see if our nam mutants represent one or more nuclear genes presumably encoding mitoribosomal proteins, and to check the allelism between our suppressors and the nam 3 suppressor mentioned above.

\section{METHODS}

Strains. These are listed in Table 1.

Nomenclature. oxil-V25 is a mutation in the mitochondrial oxil gene. It will be further referred to as oxil-, its specific wild-type allele being oxil ${ }^{+}$. box3-M2101 is a mutation in the mitochondrial box3 locus. It will be further referred to as box $3^{-}$, its wild-type allele being box $3^{+}$. Both these mutations belong to a general class of mitochondrial mit ${ }^{-}$mutations. Thus the designation mit $^{+}$refers generally to wild-type mitochondrial genomes. The mutant alleles, active as suppressors, are recessive, so they can be denoted sup ${ }^{\text {act }}$, while their standard, inactive alleles are dominant, so they can be denoted $S U P^{\text {ina }}$. Since, however, our sup ${ }^{\text {act }}$ mutants are similar to nam 3 they will be called generally nam, and their standard alleles $\mathrm{NAM}^{+}$. Prior to a final allocation of our nam mutants to

Abbreviation: EB, ethidium bromide. 

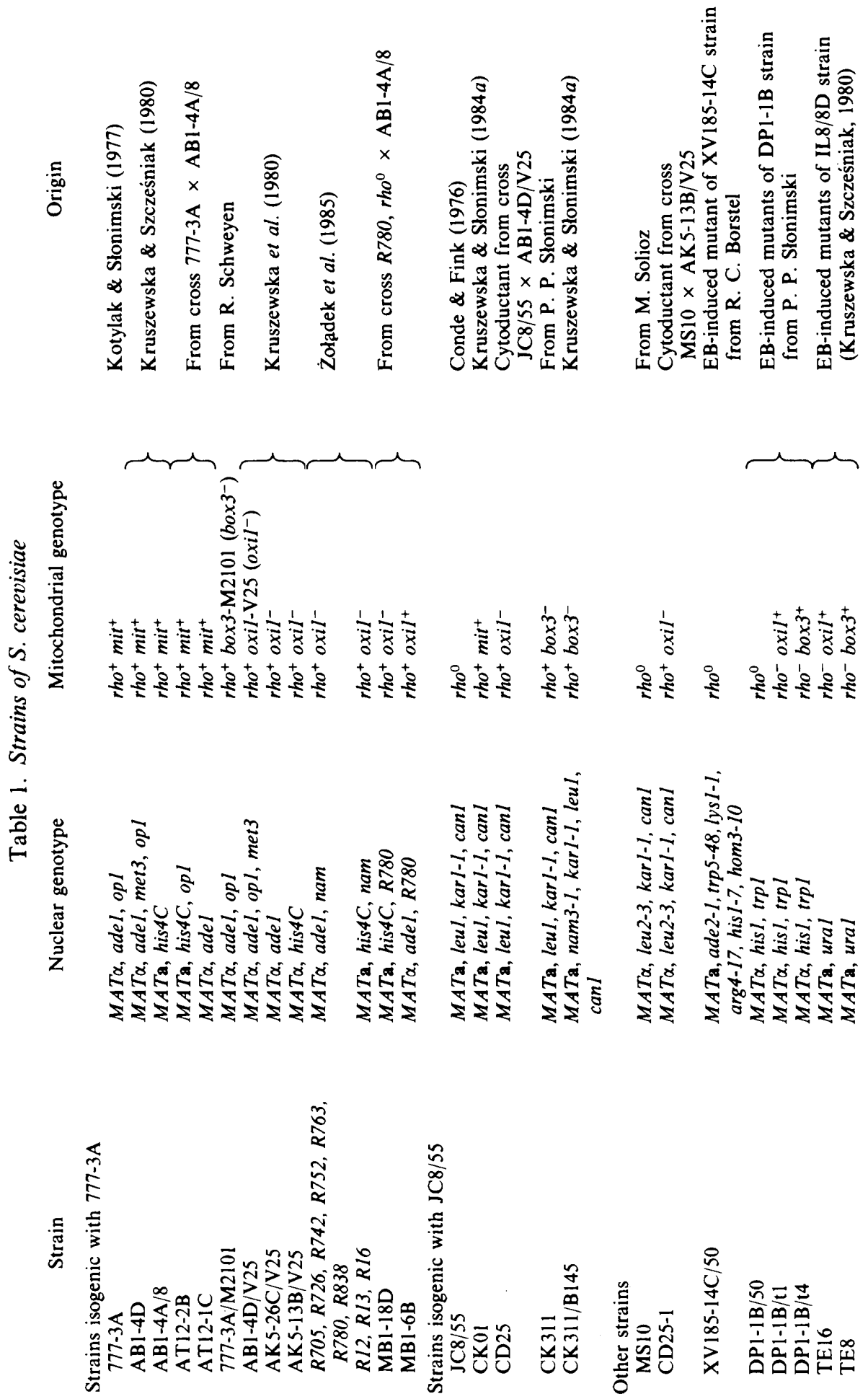
specific genes, each of the mutants will retain its isolation number, $R 12, R 13$, etc., and their respective standard, inactive alleles $-R 12^{+}, R 13^{+}$, etc.

Media. YEP $(1 \%, w / v$, peptone, $1 \%, w / v$, yeast extract), liquid or solidified with $2 \%(w / v)$ agar, was supplemented with $2 \%(\mathrm{w} / \mathrm{v})$ glucose (YEPglu) or $2 \%(\mathrm{v} / \mathrm{v})$ glycerol (YEPgly), or with $0.5 \%(\mathrm{w} / \mathrm{v})$ glucose plus $2 \%$ (v/v) glycerol (YEPdif). Minimal medium (GO, Difco Base Medium without amino acids) was supplemented with $2 \%(\mathrm{w} / \mathrm{v})$ glucose and solidified with $2 \%(\mathrm{w} / \mathrm{v})$ agar. For sporulation VB medium was used $\left(8 \cdot 2 \mathrm{~g} \mathrm{CH} \mathrm{COONa}_{3}\right.$,

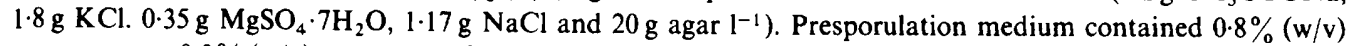
yeast extract, $0.3 \%(\mathrm{w} / \mathrm{v})$ peptone, $10 \%(\mathrm{w} / \mathrm{v})$ glucose and $2 \%(\mathrm{w} / \mathrm{v})$ agar.

Genetic methods. Apart from standard genetic methods special ones were used to establish the genotypes of tetrads. In the progenies of crosses between suppressor and standard strains $\left[\right.$ nam $_{\mathrm{x}}, \mathrm{rho}^{0} \times \mathrm{NAM}_{\mathrm{x}}^{+}, \mathrm{rho}^{+} \mathrm{mit}^{+}$(or sup ${ }^{\text {act }}, r h o^{0} \times S U P^{\text {ina }}, r h o^{+}$mit $\left.\left.^{+}\right)\right]$single-spore clones or pairs of $\mathrm{Gly}^{-}$(glycerol-negative) appeared. In order to see if they were $r h o^{-}$, they were crossed with $r h o^{0}$ testers, which served also to establish mating types. Only rho ${ }^{+} \mathrm{mit}^{+}$ spore clones gave rise to $\mathrm{Gly}^{+}$(glycerol-positive) diploids. Cytoduction was used to distinguish between spore clones carrying a mutant (nam) from standard $\left(\mathrm{NAM}^{+}\right.$) alleles (Dujardin et al., 1980). Spore clones were converted to $r h o^{\circ}$ by ethidium bromide (EB) treatment and crossed with CD25 and CD25-1 testers. Only spore clones carrying a nuclear nam mutation and mitochondria from the kar tester gave rise to Gly ${ }^{+}$cytoductants. If spore clones were prototrophic the zygotes were isolated by micromanipulation. Alternatively, spore clones to be tested were converted to $r h o^{0}$ by EB treatment and backcrossed with nam ${ }_{x}, r h o^{+}$oxil $^{-}$strains. Since the nam suppressors are recessive, only diploids homozygous for the suppressor mutation were Gly ${ }^{+}$.

In the progenies of crosses between pairs of suppressor mutations nam ${ }_{\mathrm{x}}$, rho $^{+}$oxil $^{-} \times$nam $_{\mathrm{y}}$, rho $^{+}$oxil- $^{-}$it was assumed that the $\mathrm{Gly}^{+}$spore clones had nam $\mathrm{m}_{\mathrm{x}}, \mathrm{rho}^{+}$oxil $^{-}$or nam $_{\mathrm{y}}$, rho ${ }^{+}$oxil- $^{-}$genotypes. The Gly $\mathrm{G}^{-}$spore clones could be either $r \mathrm{ho}^{-}$or $\mathrm{rho}^{+} \mathrm{oxil}^{-}$. Only the latter were restored by rho- oxil ${ }^{+}$testers, that is the respective diploids grew on YEPgly medium. These testers were used also to determine mating types. The presence of nam mutations in the Gly- spore clones was established by cytoduction or by backcrossing as above. In order to see if Gly ${ }^{+}$spore clones in the progenies of these crosses carried mitochondrial reversion of the oxil- mutation, they were crossed with $r h o^{0}$ testers. Only $r h o^{+} \mathrm{mit}^{+}$spore clones would give rise to $\mathrm{Gly}^{+}$diploids.

In progenies of the cross $O P^{+}, R 780 \times o p 1, R 780^{+}$the spore clones carrying either single $o p l$ or $R 780$ or double $o p 1, R 780$ mutations were Gly-. Their genotypes were established in two sets of tests. (a) Growth on YEPglu medium with EB; on this medium only $O P^{+}$spore clones could grow, since EB by inducing rho mutations prevented growth of strains carrying the opl mutation (Kovačova et al., 1967). (b) The Gly- spore clones were backcrossed to opl and $R 780$ strains (see Table 1); opl /opl and $R 780 / R 780$ homozygotes did not grow on glycerol medium, whereas the respective heterozygotes had Gly ${ }^{+}$phenotypes.

Other genetic methods were applied as described by Dujardin et al. (1980).

Cytochrome spectra. For spectral analysis cells were grown on YEPgal (YEP plus $2 \%$, w/v, galactose) solid medium. The cytochrome spectra of sodium dithionite-reduced cells were determined according to Claisse $e t$ al. (1970).

\section{RESULTS}

All nam mutants were recessive. Thus, directly after they had been isolated 32 mutants derived from strain AK 5-26C/V25 were replica-crossed with a set of six mutants derived from strain AK 5-13B/V25, used as testers. The results allowed a tentative subdivision of the 32 mutants into groups: (a) 13 mutants gave rise to Gly diploids in crosses with all testers, $(b) 12$ mutants gave rise to $\mathrm{Gly}^{+}$diploids in crosses with some, but not all testers, and $(c)$ the remaining seven mutants gave rise to Gly- diploids in crosses with all testers. The results did not seem to be entirely reliable, however, as some Gly- diploids from groups $(b)$ and $(c)$ showed papillary growth after 7-10 d incubation. This proved to be due to fast accumulation of rho mutations in the nam mutant strains belonging to groups $(b)$ and $(c)$. Thus, only seven mutants from AK526C/V25 (nos. $R 705, R 726, R 742, R 752, R 763, R 780$ and $R 838$; Table 1) and three from AK5$13 \mathrm{~B} / \mathrm{V} 25$ (nos. $R 12, R 13$ and $R 16$, Table 1 ) were subcloned and taken for further analysis. Even after subcloning, mitochondrial genomes in the strains carrying nam mutations were rather unstable (Table 2).

The complementation test, involving the 10 nam mutations discussed above, was repeated (Table 3). The results could be compatible with the assumption that all nam mutants tested are allelic, but two facts made this assumption doubtful. Firstly, diploid $R 13 / R 780$, oxi ${ }^{-}$was $\mathrm{Gly}^{-}$. Secondly, some combinations of nam mutants, particularly $R 12 / R 780$, oxil- , grew on YEPgly medium much better than did either of the mutants alone (respective haploids). Moreover, this 
Table 2. Percentage of rho- cells in suppressor strains

The cells were pre-grown in liquid YEPglu medium, and plated on YEPdif. The percentage of rho ${ }^{-}$cells is a mean from two experiments for all original isolates except $R 780$, tested in five experiments, for which the lowest and highest values are given. The spore clones from the MB1 cross were tested in a single experiment.

\begin{tabular}{|c|c|}
\hline Strain & $\begin{array}{c}\text { Percentage } \\
r h o^{-}\end{array}$ \\
\hline $\mathrm{AB} 1-4 \mathrm{~A} / 8$ (control) & 5.5 \\
\hline$R 12, o x i l^{-}$ & 3.5 \\
\hline RI3, oxil- & 43.5 \\
\hline$R 16$, oxil $^{-}$ & 12.5 \\
\hline R705, oxil- & 8.5 \\
\hline R726, oxil- & $36 \cdot 0$ \\
\hline R742, oxil- & 15.5 \\
\hline$R 752$, oxil $^{-}$ & 18.5 \\
\hline$R 763$, oxil $^{-}$ & $41 \cdot 0$ \\
\hline$R 780$, oxiT $^{-}$ & 24-98 \\
\hline$R 838$, oxil $^{-}$ & $28 \cdot 0$ \\
\hline $\mathrm{MB} 1-6 \mathrm{~A}, R 780^{+}, r h 0^{+}, \mathrm{mit}^{+}$ & 6.0 \\
\hline MB1-6B, $R 780^{-}, r h o^{+}, \mathrm{mit}^{+}$ & $84 \cdot 0$ \\
\hline $\mathrm{MB1}-6 \mathrm{C}, R 780^{-}, r h o^{+}, \mathrm{mit}^{+}$ & $98 \cdot 0$ \\
\hline $\mathrm{MB} 1-6 \mathrm{D}, R 780^{+}, r h o^{+}, \mathrm{mit}^{+}$ & 6.0 \\
\hline
\end{tabular}

Table 3. Results of complementation tests between suppressor mutants of the nam type

The diploids were obtained by replica-crosses, $M A T$ a strains being used as lawns. They were replicated onto YEPgly medium and incubated for $5 \mathrm{~d} .+,++,+++$, poor, good and very good growth, respectively; - , no growth; NT, not tested.

\begin{tabular}{|c|c|c|c|c|c|c|c|c|}
\hline $\begin{array}{c}\text { nam } \\
\text { mutant }\end{array}$ & $M A T \mathbf{a}$ & $R 12$ & $R 13$ & $R 16$ & $R 726$ & $R 742$ & $R 763$ & $R 780$ \\
\hline \multicolumn{9}{|l|}{$M A T \alpha:$} \\
\hline$R 13$ & & + & ++ & + & NT & NT & NT & - \\
\hline$R 705$ & & ++ & + & + & ++ & + & ++ & + \\
\hline$R 726$ & & +++ & + & + & ++ & ++ & +++ & $++t$ \\
\hline$R 742$ & & +++ & + & + & + & ++ & +++ & ++ \\
\hline$R 752$ & & + & ++ & + & ++ & ++ & + & ++ \\
\hline$R 763$ & & ++ & ++ & ++ & + & + & ++ & ++ \\
\hline$R 780$ & & +++ & - & + & ++ & ++ & ++ & + \\
\hline$R 838$ & & + & + & + & + & ++ & $++t$ & +++ \\
\hline
\end{tabular}

particular diploid had a cytochrome spectrum resembling that of the wild-type rather than of either $R 12$, oxil- or $R 780$, oxil- alone. Both these cases suggested that in heterozygotic (or heteroallelic) diploids there is some sort of interaction between mutant gene products with opposite phenotypic effects (see Discussion). The results suggested that complementation tests were not sufficient for unequivocal classification of the nam mutants as being either allelic or non-allelic. This promoted us to test their allelism in crosses.

\section{Segregation of the nam mutations, and their interaction with standard mitochondrial genomes}

Prior to analysis of pairwise crosses between different nam mutants it was necessary to determine their segregation in crosses with standard $N A M^{+}$strains. Thus, synchronous crosses nam $_{\mathrm{x}}$, oxil $^{-} \times N A M_{\mathrm{x}}^{+}, r h o^{\circ}$ were made and the zygotes transferred directly into sporulation media. Sporulation was, however, poor and among spore clones mitochondrial revertants were found. It was decided, therefore, to analyse $n a m_{\mathrm{x}}, r h o^{0} \times N A M_{\mathrm{x}}^{+}, r h o^{+}$oxil ${ }^{+}$crosses. In this way segregation of the nam mutations could be followed indirectly, and the effects, if any, of the nam mutations on standard mitochondrial genomes could be tested.

In crosses involving eight nam mutants (see Table 2, all but $R 13$ and $R 780$ mutants) all spore 


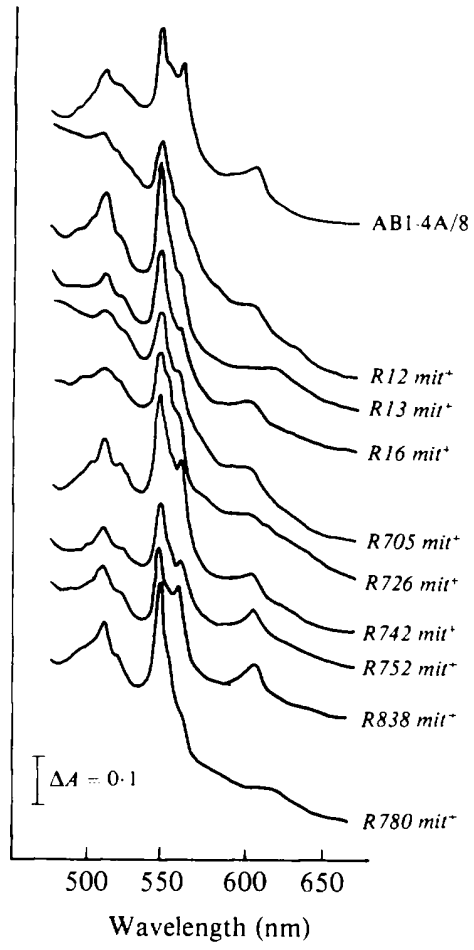

Fig. 1. Cytochrome spectra of nam ( $R$ series) $r h o^{+} m_{i t^{+}}$strains. Experimental procedure was as described in Methods.

clones grew on YEPgly equally well. The tetrads were tested for the presence of the nam mutations by cytoduction and backcrosses. Only 3-10 tetrads from each cross were tested. In all of them 2:2 segregation was found. Thus, the original isolates carried either single nam mutations, or double ones, linked with each other. In spite of good growth on YEPgly medium the cytochrome spectra of some nam $_{\mathrm{x}}, \mathrm{rho}^{+} \mathrm{mit}^{+}$strains were abnormal (Fig. 1).

Different results were found in the crosses involving $R 13$ and $R 780$. In 26 tetrads from cross $R 13, r h o^{0} \times R 13^{+}, r h o^{+} \mathrm{mit}^{+}$pairs of spore clones showed delayed and poor growth on YEPgly medium. They proved to carry the $R 13$ mutation, while spore clones growing normally on YEPgly medium carried the $R 13^{+}$allele. It can be concluded, therefore, that $R 13$ is a single mutation. It rather inefficiently suppresses the oxil- mitochondrial mutation, and affects unfavourably the functions of the normal mitochondrial genome. In 30 tetrads from cross $R 780$, $r h o^{0} \times \mathrm{R}^{2} 80^{+}$, rho $^{+} \mathrm{mit}^{+}$(MBl cross) a clear-cut 2:2 segregation into $\mathrm{Gly}^{+}: \mathrm{Gly}^{-}$spore clones was found. The $\mathrm{Gly}^{-}$spore clones were $\mathrm{rho}^{+} \mathrm{mit}^{+}$; thus their inability to grow on YEPgly medium resulted from segregation of a nuclear gene. Therefore we tested whether the original strain AK 5-26C/V25/R780 was in fact a double mutant carrying in addition to the nam supressor a pet mutation; in the the mitochondrial oxi $1^{-}$background the suppressor could mask the effect of the pet mutation, which was phenotypically expressed in the presence of the normal mitochondrial genome. To check this possibility, a number of tests were carried out. Four spore clones from a complete tetrad of the MB1 cross were analysed for $r o^{-}$accumulation (Table 2, bottom) and crossed with standard $\mathrm{R} 8 \mathrm{O}^{+}, \mathrm{rho}^{+} \mathrm{mit}^{+}$strains, and tetrads from each cross were analysed. As expected, in crosses involving $\mathrm{Gly}^{+}$spore clones all progeny were $\mathrm{Gly}^{+}$, while in crosses involving $\mathrm{Gly}^{-}$spore clones the progeny showed $2: 2$ segregation into $\mathrm{Gly}^{+}$and $\mathrm{Gly}^{-}$ spore clones. A number of spore clones from each cross was tested as described above and the results were once more as expected (see Table 4 ). Thus $R 780$ is probably a single nuclear mutation. 
Table 4. Presumed genotypes of spore clones from crosses between $R 780$ (original isolate converted to rho ${ }^{\circ}$ ) and standard strains

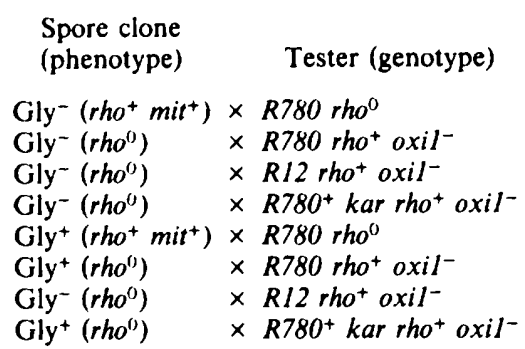

Inferred genotype of

diploid or cytoductant

$R 780 / R 780$ rho $^{+}$oxil $^{+}$

$R 780 / R 780 \mathrm{rho}^{+} \mathrm{oxil}^{-}$

R780/RI2 rho $^{+}$oxil-

Cytoductant: $R 780$ rho $^{+}$oxil-

$R 780^{+} / R 780 \mathrm{rho}^{+} \mathrm{oxil}^{+}$

$R 780^{+} / R 780$ rho $^{+}$oxil ${ }^{-}$

$R 780^{+} / R 12$ rho $^{+}$oxil $^{-}$

Cytoductant: $R 780^{+}$rho+ oxil ${ }^{-}$
Its growth on glycerol medium

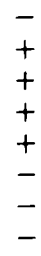

\section{Checking allelism of nam mutants in pairwise crosses}

Since complementation tests gave ambiguous results, and each nam mutant probably carried a single suppressor mutation, it was possible to proceed with testing their allelism by means of tetrad analysis. Apart from the $R 780 / R 13$ combination, which was $\mathrm{Gly}^{-}$and did not sporulate, there was no indication which pair of nam mutants was most likely to be non-allelic. Thus, instead of an extensive analysis of a few crosses, many crosses were analysed and 10-20 tetrads dissected from most of them. The crosses sporulated poorly, so that there was some danger that the preferentially sporulating cells carried mitochondrial revertants of the oxil- mutation. Tetrads from nine crosses were checked for reversion by crossing with rho ${ }^{0}$ testers. The results indicated that in none of them were mitochondrial revertants of the oxil- mutation present.

The results of the crosses can be divided roughly into three groups (Table 5). In those assigned to group A there were $4 \mathrm{Gly}^{+++}: 0 \mathrm{Gly}^{-}$tetrads only. All spore clones showed equally good growth on YEPgly medium, and therefore they carried one or another suppressor mutation segregating in the respective cross. The infrequent $r h o^{0}$ mutants also carried nam mutations.

In crosses assigned to group B, apart from infrequent $r o^{-}$spore clones, a 2:2 segregation into fast-growing and slow-growing spore clones was found. In five of a total of six such crosses, one of the parental strains was that carrying the $R / 3$ suppressor (the one which showed 2:2 segregation into well-growing and poorly-growing spore clones in crosses with the standard, $\mathrm{NAM}^{+}, \mathrm{rho}^{+} \mathrm{mit}^{+}$tester).

In crosses assigned to group $\mathrm{C}$ the phenotypes of spore clones were erratic. In some crosses involving $R 13$, in some tetrads there was $2: 2$ segregation into spore clones growing well and poorly on glycerol medium, while in other tetrads there was segregation into $2 \mathrm{Gly}^{+++}: 1 \mathrm{Gly}^{+}: 1$ Gly- spore clones. In crosses involving $R 780,2 \mathrm{Gly}^{+++}: 2 \mathrm{Gly}^{-}$segregation predominated; occasionally there were tetrads segregating into $2 \mathrm{Gly}^{+++}: 1 \mathrm{Gly}^{+}: 1 \mathrm{Gly}^{-}$. A number of spore clones from crosses involving $R 780$ were further tested as described in Methods, and their genotypes proved to be $R 780, r h o^{+}$oxil $^{-}$.

In none of the crosses enumerated in Table 5 were spores carrying the wild-type $\left(N_{A M}{ }^{+}\right)$ allele of the suppressor mutants found. Thus, either all the suppressor mutants studied here are allelic, or they result from mutations in two (or more) closely linked genes.

\section{Further analysis of $R 780$ and $R 13$ suppressors}

As shown in the preceding sections, not only all $R 780 \mathrm{rho}^{+} \mathrm{mit}^{+}$, but also the majority of R780 $\mathrm{rho}^{+}$oxil $^{-}$spore clones were $\mathrm{Gly}^{-}$. A similar but much weaker effect was exhibited by $R$ 13. The data presented earlier (Zołądek et al., 1985) show other phenotypic similarities between $R 780$ and $R 13$, the most striking being their high sensitivity to paromomycin. This prompted us to perform additional tests, mainly with $R 780$. The $R 780 / R 13$, rho ${ }^{+}$oxil- diploid was Gly ${ }^{-}$. If this was indeed due to complementation between these two mutations, then diploid $R 780 / R 13$, rho $^{+}$oxil ${ }^{+}$should be Gly+. Such a diploid was constructed, and it proved to be Gly ${ }^{-}$. After several weeks of storage in a refrigerator a small fraction of diploid cells started growth when plated on glycerol medium. The bearing of these data on the problem of complementation between $R 780$ and $R 13$ will be analysed in the Discussion. 
Table 5. Segregation in crosses nam $_{\mathrm{x}} \times$ nam $_{\mathrm{y}}$, oxil-

In most crosses few complete tetrads germinated. Since the chief purpose of the analysis was to look for the $\mathrm{NAM}^{+}$recombinants, total numbers of spores tested are given. Segregation into $\mathrm{Gly}^{+++}, \mathrm{Gly}^{+}$and $\mathrm{Gly}^{-}$(good, poor and no growth on YEPgly medium, respectively) is based on a few complete tetrads, but the presence of the three types of spore clones could be deduced from incomplete tetrads, in which three spores were viable.

\begin{tabular}{|c|c|c|c|c|c|}
\hline \multirow[b]{2}{*}{ Cross } & \multicolumn{3}{|c|}{$\begin{array}{l}\text { Segregation in } \\
\text { tetrads }\end{array}$} & \multirow{2}{*}{$\begin{array}{l}\text { No. of full } \\
\text { tetrads }\end{array}$} & \multirow{2}{*}{$\begin{array}{c}\text { Total no. of } \\
\text { spores }\end{array}$} \\
\hline & Gly ${ }^{+++}$ & Gly ${ }^{+}$ & :Gly- & & \\
\hline \multicolumn{6}{|l|}{ Group A } \\
\hline$R 838 r h o^{0} \times R 705$ oxil $^{-}$ & 4 & & 0 & 13 & 128 \\
\hline$R 838 r^{2} 0^{\circ} \times R 726$ oxil $^{-}$ & 4 & & 0 & 5 & 92 \\
\hline$R 838 r h o^{\circ} \times R 742 o_{o x i l^{-}}$ & 4 & & 0 & 13 & 126 \\
\hline$R 838 r^{2} o^{0} \times R 763$ oxil $^{-}$ & 4 & & 0 & 5 & 122 \\
\hline${ }^{*} R 838 r h 0^{0} \times R 12$ oxil $^{-}$ & 4 & & $\mathbf{0}$ & 6 & 95 \\
\hline${ }^{*} R 838 r h 0^{0} \times R 16$ oxil $^{-}$ & 4 & & 0 & 7 & 76 \\
\hline$R 705 r h 0^{\circ} \times R 726$ oxil $^{-}$ & 4 & & $\mathbf{0}$ & 19 & 112 \\
\hline$R 705 r h o^{0} \times R 763 o_{o x i l^{-}}$ & 4 & & $\mathbf{0}$ & 6 & 122 \\
\hline \multicolumn{6}{|l|}{ Group B } \\
\hline$R 838 r h 0^{\circ} \times R 752$ oxil $^{-}$ & 2 & 2 & & 12 & 117 \\
\hline${ }^{*} R 13 r h o^{\circ} \times R 742 o^{o x i 1^{-}}$ & 2 & 2 & & 7 & 89 \\
\hline RI3 rho ${ }^{\circ} \times R 763$ oxil $^{-}$ & 2 & 2 & & 3 & 61 \\
\hline${ }^{*} R 13 r h o^{0} \times R 838$ oxil $^{-}$ & 2 & 2 & & 5 & 36 \\
\hline${ }^{*} R 13 r^{\circ} 0^{0} \times R 705$ oxil - & 2 & 2 & & 5 & 50 \\
\hline${ }^{*} R 12$ oxil $^{-} \times \quad R 742$ oxil $^{-}$ & 2 & 2 & & 4 & 162 \\
\hline \multicolumn{6}{|l|}{ Group C } \\
\hline \multirow[t]{2}{*}{ R13 rho ${ }^{\circ} \times R 726$ oxil $^{-}$} & 2 & 2 & & 3 & \\
\hline & 2 & 1 & 1 & 1 & 47 \\
\hline \multirow[t]{2}{*}{${ }^{*} R 13 r h o^{0} \times R 12 \mathrm{oxil}^{-}$} & 2 & 2 & & 2 & 0 \\
\hline & 2 & 2 & 1 & 3 & 90 \\
\hline \multirow[t]{3}{*}{$R 780$ oxil $^{-} \times R 705 r h o^{0}$} & 2 & 2 & & 1 & \\
\hline & 2 & 1 & 1 & 2 & 207 \\
\hline & 2 & & 2 & 10 & \\
\hline \multirow[t]{2}{*}{$R 780$ oxil $^{-} \times R 12 r h o^{\circ}$} & 2 & 1 & 1 & $2 \dagger$ & 56 \\
\hline & 2 & & 2 & 1 & 30 \\
\hline$R 780 \mathrm{oxil}^{-} \times R 16 r h o^{\circ}$ & 2 & & $2+$ & 0 & 33 \\
\hline
\end{tabular}

* Spore clones from these crosses were crossed with $r h 0^{0}$ testers to check mitochondrial reversion. $\uparrow$ Segregation is estimated on the basis of incomplete tetrads only.

It turned out that $R 780, r h o^{+}$oxil ${ }^{+}$strains can also give rise to cells with the $\mathrm{Gly}^{+}$phenotype. Such cells occurred with a frequency of about $1 \times 10^{-4}$. Further tests showed that the adapted spore clones still carried the $R 780$ mutation and the standard mitochondrial genome. As already shown (Table 2) all spore clones carrying the $R 780$ suppressor abundantly accumulate rho ${ }^{-}$ mutations. In order to obtain a strain in which all viable cells would remain $r$ t $^{+}$the strain MB118D R780, rho ${ }^{+}$mit $^{+}$was crossed with 777-3A opl, rho ${ }^{+}$mit $^{+}$: since opl, rho $o^{-}$cells are inviable (Kovačova et al., 1967) it was expected that double mutants $R 780$, opl would remain $r h o^{+}$. In most tetrads all spores germinated, but many single ones or pairs of spore clones formed minute colonies which failed to grow further. Since both opI and $R 780$ mutations prevent growth on YEPgly medium it was possible to distinguish parental ditype (PD), non-parental ditype (NPD) and tetratype $(\mathrm{T})$ tetrads. The minute inviable spore clones appeared only in the presumed NPD and $\mathrm{T}$ tetrads. Out of a total of 21 tetrads there are 2 PD, 7 NPD and $12 \mathrm{~T}$. Thus, the total of presumed double mutant $o p 1, R 780$ spores was $26 ; 14$ of them formed minute colonies which failed to grow further, and 12 spore clones survived. The genotypes of a number of the surviving spore clones were established by backcrossing to opl and $R 780$ testers. The results were as expected. Growth of the $o p 1, R 780$ double mutant was strongly impaired even in glucose medium. 
It can be concluded, therefore, that the $R 780$ mutation, along with its suppression of $o x i 1^{-}$and other mit $^{-}$mutations, interferes with mitochondrial functions, renders mitochondrial genomes unstable and thus causes selective loss of viability in spore clones of double mutants op $1, R 780$.

\section{Complementation tests and analysis of crosses with nam3-1}

Complementation tests involved crosses of nam3-1, rho $0^{\circ}$ with $R 705, R 726, R 742, R 752, R 763$, $R 780$ and $R 838$, all rho ${ }^{+}$oxil $^{-}$(V25), and reciprocal ones, nam3-1, rho ${ }^{+}$box3- (M2101) with $R 705, R 726, R 742, R 752$ and $R 838$, all $r h o^{\circ}$. Both sets of diploids were Gly ${ }^{+}$. Thus there is no complementation between $R 705-R 838$, and nam3-1 suppressor mutations. Since, however, complementation tests proved to be unreliable in establishing allelism of the suppressors, crosses were analysed: $R 705, M A T \alpha$, adel, $r h o^{\circ}$ (initial isolate converted to rho by EB treatment) $\times$ CK311/B145/1 MATa, nam3-1, rho box3- (MB16) and R705, MATa, adel, rho $^{+}$oxil $^{-} \times$CK311/B145/1 MATa, nam3-1, rho ${ }^{\circ}$ (MB17), and respectively, 35 and 28 tetrads were dissected. In both crosses three types of tetrads were found $4 \mathrm{Gly}^{+}: 0 \mathrm{Gly}^{-}, 2 \mathrm{Gly}^{+}: 2 \mathrm{Gly}^{-}$ and $3 \mathrm{Gly}^{+}: 1 \mathrm{Gly}^{-}$. Fifteen $\mathrm{Gly}^{-}$spore clones from the MB16 cross and three from the MB17 cross proved to be $r o^{-}$, and the respective tetrads were discarded. All remaining $\mathrm{Gly}^{-}$spore clones were tentatively classified as being rho $^{+}$oxil $^{-}$(or box $3^{-}$) and carrying inactive alleles of both suppressor mutations. The proportion of tetrads from both crosses was: PD 11, NPD 9, T35.

In order to see if indeed some $\mathrm{Gly}^{+}$spore clones were double mutants nam3-1, R705, while all $\mathrm{Gly}^{-}$ones carried both respective wild-type alleles, further tests were performed. Three complete NPD and two T tetrads were tested in crosses with standard $\mathrm{R}^{2} 05^{+}, \mathrm{NAM}^{+}$, rho ${ }^{\circ}$ strains, and 16 spore clones from other tetrads - by cytoduction. Their genotypes were as expected. Growth on YEPgly medium of diploids with the cis configuration of the suppressors $R 705$, nam3-1 $\times R^{2} 705^{+}, N A M 3^{+}$was poorer than those of the trans configuration $R 705$, $N A M 3^{+} \times R 705^{+}$, nam3-1. The difference could be due to the different genetic background of the strains: the double mutants were progeny of a cross between the strain related to $777-3 \mathrm{~A}$ and to JC8.

It can be concluded, therefore, that in spite of the absence of complementation, our suppressor mutants of the R series are non-allelic with nam3-1. The proportion of PD:NPD:T tetrads is close to $1: 1: 4$. This indicates that the respective genes are unlinked either with each other or with their respective centromeres.

\section{DISCUSSION}

The main purpose of the present work was to test allelism of 10 nuclear suppressors of the mitochondrial mutation oxi1- (Żoładek et al., 1985) and to find if they were allelic with nam3-1 isolated as a revertant of the mitochondrial mutation box3- (M2101), but resembling in many respects our suppressors (Kruszewska \& Slonimski, 1984a, $b$ ). The allelism was studied by complementation tests and by analysis of pairwise crosses of the type nam $/$ nam $_{\mathrm{y}}$, rho $^{+}$mit $^{-}$. There is a striking difference between the results of the two tests. Complementation tests interpreted formally indicate that all suppressors, including nam3-1, but $R 780$ and $R 13$, are allelic. $R 13$ and $R 780$ could be formally assigned as showing intragenic complementation. If this were the case, then the $R 780 / R 13, r h o^{+}$mit $^{+}$diploid should grow on YEPgly medium, but in fact this diploid was $\mathrm{Gly}^{-}$.

On the other hand, the results of tetrad analysis show clearly that the 10 suppressors studied by us are closely linked, and therefore they may be allelic, but one of our mutants $(R 705)$ and the nam3-1 suppressor segregate independently. The strains used by us to isolate nam mutants of the $\mathrm{R}$ series are not isonuclear with that used by Kruszewska \& Słonimski $(1984 a, b)$ to isolate nam3-1. Thus, it is possible that our nam mutations as well as nam3-1 occurred in the same gene, which in these strains is located on different chromosomes as a result of a translocation. In this case in NPD tetrads pairs of $\mathrm{Gly}^{+}$spores, and in T tetrads single spores would carry a double dose of identical genes, one with $R 705$ and the other with the nam3-1 mutation, while pairs and single $\mathrm{Gly}^{-}$spores, respectively, would lack entirely a single mitoribosomal protein. Such strains need 
not apparently have inactive mitoribosomes (De Wilde et al., 1977). It seems improbable, however, that the two strains differ only in translocation of a single gene. All the data on genetic maps make such a possibility unlikely. If, on the other hand, the translocation involved a longer chromosomal segment then the crosses we analysed would be heterozygous in respect of the translocation. Such crosses show a decreased spore viability (Perkins \& Barry, 1977), whereas in the crosses we analysed spore viability was over $90 \%$.

Thus, a different interpretation of our results is indicated. Indirect evidence (Kruszewska \& Słonimski, 1984 a, $b$; Żoladek et al., 1985) suggests that the nam mutations under discussion act as suppressors of the mitochondrial mutations by changing the conformation of the small mitoribosomal subunit and thus decreasing the fidelity of translation. The structure of yeast mitoribosomes is not known in detail, but it can be supposed that they are not essentially unlike those of $E$. coli. In the small subunit of $E$. coli ribosomes there is only a single molecule of each ribosomal protein per particle (Hardy, 1975). This seems to be true also for the small mitoribosomal subunit of yeast (Terpstra et al., 1979). Since intragenic complementation is due to interaction between two or more polypeptides specified by allelic genes, it cannot occur at all between the allelic genes encoding mitoribosomal proteins. Dominance of standard over mutant alleles (the latter being active as suppressors) can be due to a higher affinity of the former for ribosome assembly (see Ishiguro et al., 1981 ; Green \& Kurland, 1971, cited after Olsson, 1979) and/or to faster degradation of the protein molecules spicified by mutant genes (Olsson \& Isacsson, 1979). In double heterozygotes there are two types of altered mitoribosomal proteins specified respectively by $R 705$ and nam 3-1 mutani genes, and two standard proteins specified by their respective standard alleles. Thus, in contrast to single heterozygotes $R 705 / R 705^{+}$and

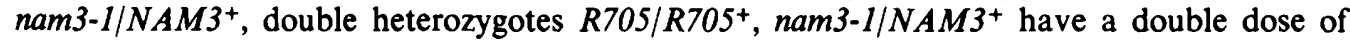
mutant genes. This increases twofold the probability of incorporation into the ribosomes of one or other of the mutant proteins, as compared with the single heterozygotes. This probability can be further increased if there is a limited number of sites involved in transport of the mitoribosomal proteins into mitochondria. Thus, in such heterozygotes the population of mitoribosomes can be mixed : most particles will carry both standard proteins, but a fraction of them will carry one or another altered protein. Even a small fraction of mitoribosomes with a decreased translation fidelity can be sufficient to suppress mit ${ }^{-}$mutations; as is apparently the case with the semidominant omnipotent cytosolic suppressor SUP46 (Ishiguro et al., 1981). Bolotin-Fukuhara (1979) found complementation between nuclear suppressors of mitochondrial $C^{\mathrm{R}}$ mutation: the respective diploids were $C^{\mathrm{R}}$ rather than $C^{\mathrm{S}}$. These data need not disagree with ours. The suppressors could be non-allelic, as the author stated, but the double heterozygotes could have a mixed population of mitoribosomes : resistant and sensitive to chloramphenicol, so that the cells could grow on the drug-containing medium, as we assume is the case with growth on YEPgly medium of $R 705 / \mathrm{RTOS}^{+}, \mathrm{NAM}^{+} /$nam3-1 oxil- heterozygotes.

This interpretation of our results does not accord with the behaviour of the omnipotent suppressors acting in cytoplasmic protein synthesis in yeast. The majority of these suppressors are recessive and can be classified as being allelic to either $\sup 35(\sup Q, \operatorname{sup1})$ or $\sup 45(\sup P, \sup 2)$ loci (Hawthorne \& Leupold, 1974; Gerlach, 1975; recent review: Surguchov et al., 1984). This difference may be connected with different compartmentation conditions between the cytosolic and mitochondrial ribosome assembly: in the latter, transport of ribosomal proteins into mitochondria may be a limiting factor in the assembly of ribosomes.

It should be mentioned that there is at least one well-documented case of absence of complementation between suppressor mutations mapping $20 \mathrm{cM}$ apart (Lewis, 1961).

If the above hypothesis is correct then the behaviour of $R 13$ and $R 780$ mutations can be explained by assuming that the respective mutant genes are allelic; the altered proteins interfere with each other during ribosome assembly so that few if any functional particles are formed. This results in a Gly ${ }^{-}$phenotype of both $R 13 / R 780$, rho ${ }^{+}$oxil $^{-}$and $R 13 / R 780$, rho ${ }^{+}$oxil $^{+}$ diploids. On the other hand, the good growth on YEPgly medium and nearly normal cytochrome spectrum of the $R 12 / R 780$, rho+ $\mathrm{oxil}^{-}$diploid may indicate that these two mutations are in fact non-allelic.

The other properties of the $R 780$ suppressor can be interpreted as follows. In strains carrying 
$R 780$ the assembly or functioning of mitoribosomes is abnormal. This leads to the accumulation of rho- mutations. The same effect is observed in strains carrying mitochondrial mutations affecting mitochondrial protein synthesis (see e.g. Bolotin-Fukuhara, 1979; Julou \& BolotinFukuhara, 1982). This effect of $R 780$ is particularly strong in spores, so that upon germination the spore clones are unable to respire. This results in cell lethality of the double mutants $R 780$, $o p 1$. Spores carrying a deletion mutation nam $2-\Delta 8$ and $o p 1$ were also inviable (Labouesse $e t$ al., 1985). Some cytosolic suppressors show a similar effect on spore viability (Gerlach, 1975).

Most of the above interpretations can be tested by a thorough biochemical analysis of mitoribosomes from strains carrying suppressor mutations. Preliminary experiments are in progress.

This work was supported by The Polish Academy of Sciences under Project 09.7.2. The authors are grateful to Dr A. Kruszewska for gift of strains and for advice in the course of this work.

\section{REFERENCES}

Bolotin-Fukuhara, M. (1979). Mitochondrial and nuclear mutations that affect the biogenesis of the mitochondrial ribosomes of yeast. Molecular and General Genetics 177, 39-46.

Claisse, M. L., Péré-Aubert, G., Clavilier, L. \& SkONIMSKI, P. P. (1970). Methode d'estimation de la concentration des cytochromes dans les cellules entieres de levure. European Journal of Biochemistry 16, 430-438.

Conde, J. \& FINK, G. (1976). A mutant of Saccharomyces cerevisiae defective for nuclear fusion. Proceedings of the National Academy of Sciences of the United States of America 73, 3651-3655.

De Wilde, M., Cabezon, T., Herzog, A. \& Bollen, A. (1977). Apports de la génétique a la connaissance du ribosome bacterien. Biochimie 59, 125-140.

Dujardin, G., Pajot, P., Groudinsky, O. \& SlonIMSKI, P. P. (1980). Long range control circuits within mitochondria and between nucleus and mitochondria. I. Methodology and phenomenology of suppressors. Molecular and General Genetics 179, 469-482.

GerLACH, W. L. (1975). Genetic properties of some amber-ochre supersuppressors in Saccharomyces cerevisiae. Molecular and General Genetics 138, 58-63.

Green, M. \& Kurland, G. C. (1971). Mutant ribosomal protein with defective RNA binding site. Nature New Biology 234, 273-275.

HARDY, S. J. S. (1975). The stoichiometry of the ribosomal proteins of Escherichia coli. Molecular and General Genetics 140, 253-274.

Hawthorne, D. C. \& Leupold, U. (1974). Suppressors in yeast. Current Topics in Microbiology and Immunology 64, 1-47.

ishiguro, J., ONo, B., Masurekar, C. S., MclaughLIN, M. \& SHERMAN, F. (1981). Altered ribosomal protein S11 from SUP46 suppressor of yeast. Journal of Molecular Biology 147, 391-397.

Julou, C. \& Bolotin-Fukuhara, M. (1982). Genetics of mitochondrial ribosomes of yeast: mitochondrial lethality of a double mutant carrying two mutations of the 21S ribosomal RNA gene. Molecular and General Genetics 188, 256-260.

Ková̌ova, V., Irmlerova, J. \& Kovac, L. (1967). Oxidative phosphorylation in yeast. IV. Combination of a nuclear mutation affecting oxidative phosphorylation with cytoplasmic mutation to respiration deficiency. Biochimica et biophysica acta 162, 157-163.

KotYlak, Z. \& Slonimski, P. P. (1977). Fine structure genetic map of the mitochondrial DNA region controlling coenzyme QH2-cytochrome $c$ reductase. In Genetics and Biogenesis of Mitochondria, pp. 161172. Edited by W. Bandlow, R. J. Schweyen, K. Wolf \& F. Kaudewitz. Berlin: Walter de Gruyter.

Kruszewska, A. \& Slonmski, P. P. (1984a). Mitochondrial and nuclear mitoribosomal suppressors that enable misreading of ochre codons in yeast mitochondria. I. Isolation, localization and allelism of suppressors. Current Genetics 9, 1-10.

KruszewsKa, A. \& Slonimski, P. P. (1984b). Mitochondrial and nuclear suppressors that enable misreading of ochre codons in yeast mitochondria. II. Specificity and extent of suppressor action. Current Genetics 9, 11-19.

KruszewsKa, A. \& SzcześñaK, B. (1980). Construction of isomitochondrial and isonuclear strains for recombinational analysis of mitochondrial loci in Saccharomyces cerevisiae. Genetical Research 35, 225229.

Kruszewska, A., Szcześniak, B. \& Clansse, M. L. (1980). Recombinational analysis of oxil mutants and preliminary analysis of their translation products in Saccharomyces cerevisiae. Current Genetics 2, 45-51.

Labouesse, M., Dujardin, G. \& Slonimski, P. P. (1985). The yeast nuclear gene $N A M 2$ is essential for mitochondrial DNA integrity and can cure a mitochondrial RNA-maturase deficiency. Cell 41, 133-143.

LEWIS, D. (1961). Genetical analysis of methionine suppressors in Coprinus. Genetical Research 2, 141155.

OLsson, M. O. (1979). Analysis of rspD mutations in Escherichia coli. II. Physiology of some representative mutants. Molecular and General Genetics 169, 259-269.

OLsson, M. O. \& IsAcsson, L. A. (1979). Analysis of $r s p D$ mutations in Escherichia coli. III. Effects of $r p s D$ mutations on expression of some ribosomal protein genes. Molecular and General Genetics 169 , 271-278. 
Ono, B., Stewart, J. W. \& Sherman, F. (1981). Serine insertion caused by the ribosomal suppressor SUP46 in yeast. Joumal of Molecular Biology 147, 373-379. PERkINS, D. D. \& BARRY, E. G. (1977). The cytogenetics of Neurospora. Advances in Genetics 19, 133-285. Surguchov, A. P., Smirnov, V. N., Ter-Avanesan, M. D. \& INGe-Vechmontov, S. G. (1984). Ribosomal suppression in eukaryotes. Physicochemical Biology Reviews 4, 147-205.
Terpstra, P., Zanders, E. \& Butow, R. A. (1979). The association of varl with the $38 \mathrm{~S}$ mitochondrial ribosomal subunit in yeast. Journal of Biological Chemistry 254, 12653-12661.

ŹolADEx, T., Boguta, M. \& PUTRAMENT, A. (1985). Nuclear suppressors of mitochondrial mutation oxilV25 in Saccharomyces cerevisiae. I. Phenotypes of some suppressors. Current Genetics 9, 427-433. 